\title{
THE DUAL WEIERSTRASS-LAGUERRE TRANSFORM
}

\author{
DEBORAH TEPPER HAIMO ${ }^{1}$
}

\begin{abstract}
An inversion algorithm is derived for the dual Weierstrass-Laguerre transform $\int_{0}^{\infty} g_{\alpha}(x, y ; 1) \varphi(y) y^{\alpha} e^{-y} /(\alpha+1) d y$, where the function $g_{\alpha}(x, y, t)$ is associated with the source solution of the Laguerre differential heat equation $x u_{x x}(x, t)=(\alpha+1-x) u_{x}(x, t)=u_{t}(x, t)$. Correspondingly, sufficient conditions are established for a function to be represented by a Weierstrass-Laguerre Stieltjes transform $\int_{0}^{\infty} g_{\alpha}(x, y ; 1) d \beta(y)$ of a nondecreasing function $\beta$.
\end{abstract}

1. Introduction. Integral transforms whose kernels are functions associated with the fundamental solutions of heat equations of various forms have been studied extensively. The author has investigated, in particular, transforms related to the generalized, the Laguerre differential, and the Laguerre difference heat equations, deriving results parallel to those for the classical heat equation developed primarily by D. V. Widder.

Continuing the project initiated in a previous work, the author seeks an inversion and representation theory for the dual Weierstrass-Laguerre transform analogous to that developed for the Weierstrass transform [8], the Weierstrass-Hankel transform [1], and the Weierstrass-Laguerre transform [4].

2. Preliminary results. Let $\alpha \geqslant 0$ and let $L_{n}^{\alpha}(x)$ denote the Laguerre polynomial of degree $n$. The Laguerre transform $f^{\wedge}(x)$ of a real function $f(n)$, defined for $n=0,1, \ldots$, is given by

$$
\hat{f^{\wedge}}(x)=\sum_{n=0}^{\infty} L_{n}^{\alpha}(x) f(n) \rho_{\alpha}(n)
$$

with

$$
\rho_{\alpha}(n)=\frac{n !}{\Gamma(n+\alpha+1)} .
$$

By inversion of the Laguerre transform, we have

$$
f(n)=\int_{0}^{\infty} L_{n}^{\alpha}(x) f^{\wedge}(x) d \Omega_{\alpha}(\dot{x})
$$

where

$$
d \Omega_{\alpha}(x)=x^{\alpha} e^{-x} d x
$$

Received by the editors July 20,1984, Inversion of the dual Weierstrass-Laguerre transform presented at AMS San Luis Obispo meeting November 11-12, 1983 and Representation for the dual Weierstrass-Laguerre transform presented at AMS Anaheim meeting January 9-13, 1985.

1980 Mathematics Subject Classification. Primary 44A15, 35C15, 35K05; Secondary 33A40, 33A65.

${ }^{1}$ The research was supported in part by a University of Missouri-St. Louis 1984 summer grant. 
We define the Laguerre differential operator $\nabla_{x}$ by

$$
\nabla_{x} f(x)=x f^{\prime \prime}(x)+(\alpha+1-x) f^{\prime}(x),
$$

and note that

$$
\nabla_{x} L_{n}^{\alpha}(x)=-n L_{n}^{\alpha}(x)
$$

and

$$
\left(\nabla_{x}+2 x \frac{\partial}{\partial x}\right) L_{n}^{\alpha}(-x)=L_{n}^{\alpha}(-x) .
$$

The Laguerre differential heat equation is defined by

$$
\nabla_{x} u(x, t)=\frac{\partial}{\partial t} u(x, t) .
$$

We denote by $H$ the class of all $C^{2}$ solutions of (2.8) and refer to a member of $H$ as a dual Laguerre temperature.

The fundamental solution of $(2.8)$ is the function

$$
g_{\alpha}(x, t)=\sum_{n=0}^{\infty} e^{-n t} L_{n}^{\alpha}(x), \quad t>0 .
$$

As in [5], we obtain the associated fundamental solution basic to our theory by introducing the function

$$
d(x, y, z)=\sum_{n=0}^{\infty} L_{n}^{\alpha}(x) L_{n}^{\alpha}(y) L_{n}^{\alpha}(z)\left[\Gamma(\alpha+1) \rho_{\alpha}(n)\right]^{2} .
$$

We have that

$$
\begin{gathered}
\int_{0}^{\infty} L_{n}^{\alpha}(z) d(x, y, z) d \Lambda_{\alpha}(z)=L_{n}^{\alpha}(x) L_{n}^{\alpha}(y)\left[\Gamma(\alpha+1) \rho_{\alpha}(n)\right], \\
\Lambda_{\alpha}(z)=\Omega_{\alpha}(z) / \Gamma(\alpha+1),
\end{gathered}
$$

and

$$
\int_{0}^{\infty} d(x, y, z) d \Lambda_{\alpha}(z)=1 .
$$

The associated function $f(x, y)$ of a function $f(x)$ defined on $[0, \infty)$ is given by

$$
f(x, y)=\int_{0}^{\infty} f(z) d(x, y, z) d \Lambda_{\alpha}(z)
$$

whenever the integral converges.

The function associated with the fundamental solution (2.8) as derived in [5] is

$$
\begin{aligned}
g_{\alpha}(x, y ; t) & =\int_{0}^{\infty} g_{\alpha}(z ; t) d(x, y, z) d \Lambda_{\alpha}(z) \\
& =\sum_{n=0}^{\infty} e^{-n t} L_{n}^{\alpha}(x) L_{n}^{\alpha}(y)\left[\Gamma(\alpha+1) \rho_{\alpha}(n)\right] \\
& =\left(\frac{e^{t}}{e^{t}-1}\right)^{\alpha+1} \exp \left(-\frac{x+y}{e^{t}-1}\right) I_{\alpha}\left(\frac{2 \sqrt{x y e^{t}}}{e^{t}-1}\right),
\end{aligned}
$$


where $\mathscr{I}_{\alpha}(z)=2^{\alpha} \Gamma(\alpha+1) z^{-\alpha} I_{\alpha}(z), I_{\alpha}(z)$ being the Bessel function of imaginary argument of order $\alpha$.

On appealing to (2.11) and using a straightforward induction argument, we establish that the function associated with the monomial $x^{n}$ is

$$
\begin{aligned}
\int_{0}^{\infty} z^{n} d & (x, y, z) d \Lambda_{\alpha}(z) \\
& =\Gamma(\alpha+1) \Gamma(n+\alpha+1) \sum_{k=0}^{n}(-1)^{k}\left(\begin{array}{l}
n \\
k
\end{array}\right) L_{k}^{\alpha}(x) L_{k}^{\alpha}(y)\left[\rho_{\alpha}(k)\right]^{2} .
\end{aligned}
$$

Associated functions satisfy the following readily established properties:

$$
\begin{aligned}
\text { (i) } & f(x, 0)=f(x), \\
\text { (ii) } & f(x, y)=f(y, x), \\
\text { (iii) } & \nabla_{x} f(x, y)=\nabla_{y} f(x, y), \\
\text { (iv) } & \nabla_{x} f(x,-y)=-\left(\nabla_{y}+2 y \frac{\partial}{\partial y}\right) f(x,-y) .
\end{aligned}
$$

Taking the function $g_{\alpha}(x, y ; t)$ as the kernel, we have the dual Poisson-Laguerre transform of a function $\phi$ defined on $[0, \infty)$ is given by

$$
u(x, t)=\int_{0}^{\infty} g_{\alpha}(x, y ; t) \phi(y) d \Lambda_{\alpha}(y), \quad t>0,
$$

whenever the integral converges. The dual Poisson-Laguerre-Stieltjes transform of a function $\beta$ of bounded variation is given by

$$
u(x, t)=\int_{0}^{\infty} g_{\alpha}(x, y ; t) d \beta(y), \quad t>0 .
$$

Our primary interest will be centered on the dual Weierstrass-Laguerre transform

$$
f(x)=\int_{0}^{\infty} g_{\alpha}(x, y ; 1) \phi(y) d \Lambda_{\alpha}(y)
$$

or its Stieltjes form

$$
f(x)=\int_{0}^{\infty} g_{\alpha}(x, y ; 1) d \beta(y) .
$$

As was established in [5], the dual Poisson-Laguerre tranforms (2.17) and (2.18) represent, within the regions of convergence of the integrals, dual Laguerre temperatures; in the case of (2.17), with initial temperature $u\left(x, 0^{+}\right)=\phi(x)$. The dual Weierstrass-Laguerre transforms (2.19) and (2.20) may thus be interpreted as dual Laguerre temperatures at constant time $t=1$. For (2.19), the particular choice of the constant, however, results in no loss of generality, since in replacing $x$ by $x\left(e^{t}-1\right) /(e-1)$ and $y$ by $y\left(e^{t+1}-e\right) /\left(e^{t+1}-e^{t}\right)$, we note that the transform (2.17) becomes

$$
u\left(x \frac{e^{t}-1}{e-1}, t\right)=\int_{0}^{\infty} g_{\alpha}(x, y ; 1) \phi\left(\frac{1-e^{-t}}{1-e^{-1}} y\right) d \Lambda_{\alpha}(y),
$$

the dual Weierstrass-Laguerre transform of $\phi\left(\left(1-e^{-t}\right) /\left(1-e^{-1}\right) x\right)$. 
3. Properties of the kernel $g_{\alpha}(x, y ; t)$. From its explicit representation (2.14) it is clear that the associated function $g_{\alpha}(x, y ; t)$ is defined for all real values of the variables $x, y$ and, in particular, we have

$$
g_{\alpha}(-x, y ; t)=\left(\frac{e^{t}}{e^{t}-1}\right)^{\alpha+1} \exp \left(\frac{x-y}{e^{t}-1}\right) \mathscr{J}_{\alpha}\left(\frac{2 \sqrt{x y e^{t}}}{e^{t}-1}\right)
$$

where

$$
\mathscr{J}_{\alpha}(z)=2^{\alpha} \Gamma(\alpha+1) z^{-\alpha} J_{\alpha}(z),
$$

$J_{\alpha}(z)$ being the ordinary Bessel function of order $\alpha$.

Since

$$
\mathscr{J}_{\alpha}^{\prime}(z)=-\frac{z}{2(\alpha+1)} \mathscr{J}_{\alpha+1}(z)
$$

we also have that

$$
\frac{\partial}{\partial y} g_{\alpha}(x, y ; t)=\frac{1}{e^{t}-1}\left[\frac{x}{\alpha+1} g_{\alpha+1}(x, y ; t)-g_{\alpha}(x, y ; t)\right] \text {. }
$$

In [5], we established the following semigroup property for the kernel.

THEOREM 3.1. For $t_{1}, t_{2}>0$,

$$
\int_{0}^{\infty} g_{\alpha}\left(x, z ; t_{1}\right) g_{\alpha}\left(z, y ; t_{2}\right) d \Lambda_{\alpha}(z)=g_{\alpha}\left(x, y ; t_{1}+t_{2}\right) .
$$

A related result, proved by using the formulation (3.1) for the kernel function and evaluating the resulting integral, is the following.

THEOREM 3.2. For $0<t_{1}<t_{2}$,

$$
\int_{0}^{\infty} g_{\alpha}\left(-x, z ; t_{1}\right) g_{\alpha}\left(-z, y ; t_{2}\right)\left(e^{-t_{1}}\right)^{\alpha+1} e^{x+z} d \Lambda_{\alpha}(z)=g_{\alpha}\left(x, y ; t_{2}-t_{1}\right) \text {. }
$$

Proof. Using (3.1) for the kernel functions and changing the variable $z$ to $z^{2}$, we find, on simplifying, that the integral in (3.6) becomes

$$
\begin{aligned}
2 \Gamma(\alpha+1)\left(\frac{e^{t_{1}}}{x y}\right)^{\alpha / 2} \frac{\left(e^{t_{2}}\right)^{\alpha / 2+1}}{\left(e^{t_{1}}-1\right)\left(e^{t_{2}}-1\right)} \exp \left(\frac{e^{t_{1}}}{e^{t_{1}}-1} x-\frac{y}{e^{t_{2}}-1}\right) \\
\cdot \int_{0}^{\infty} \exp \left(-\frac{e^{t_{2}}-e^{t_{1}}}{\left(e^{t_{1}}-1\right)\left(e^{t_{2}}-1\right)} z^{2}\right) J_{\alpha}\left(\frac{2 \sqrt{x e^{t_{1}}}}{e^{t_{1}}-1} z\right) J_{\alpha}\left(\frac{2 \sqrt{y e^{t_{2}}}}{e^{t_{2}}-1} z\right) z d z .
\end{aligned}
$$

Appealing to 8.11(23) of [7], we have, again on simplifying, that the left-hand side of (3.6) is

$$
\left(\frac{e^{t_{2}-t_{1}}}{e^{t_{2}-t_{1}}-1}\right)^{\alpha+1} \exp \left(-\frac{x+y}{e^{t_{2}-t_{1}}-1}\right) 2^{\alpha} \Gamma(\alpha+1)\left(\frac{2 \sqrt{x y e^{t_{2}-t_{1}}}}{e^{t_{2}-t_{1}}-1}\right)^{-\alpha} I_{\alpha}\left(\frac{2 \sqrt{x y e^{t_{2}-t_{1}}}}{e^{t_{2}-t_{1}}-1}\right),
$$

which, by (2.14), is the right-hand side of (3.6). 
By inversion of the series representation (2.14), or by direct evaluation of the integral, we have that

$$
e^{-n t} L_{n}^{\alpha}(x)=\int_{0}^{\infty} g_{\alpha}(x, y ; t) L_{n}^{\alpha}(y) d \Lambda_{\alpha}(y) .
$$

On the other hand, a straightforward computation of the integral involved yields the equation

$$
e^{n t} L_{n}^{\alpha}(x)=\int_{0}^{\infty} e^{-t(\alpha+1)} e^{x+y} g_{\alpha}(-x, y ; t) L_{n}^{\alpha}(-y) d \Lambda_{\alpha}(y),
$$

for, using (3.1), we note that the right-hand side of (3.8) is

$$
\frac{\left(x e^{t}\right)^{-\alpha / 2}}{e^{t}-1} \exp \left(\frac{x e^{t}}{e^{t}-1}\right) \int_{0}^{\infty} \exp \left(-\frac{y}{e^{t}-1}\right) J_{\alpha}\left(\frac{2 \sqrt{x y e^{t}}}{e^{t}-1}\right) y^{\alpha / 2} L_{n}^{\alpha}(-y) d y .
$$

With a change of variable, this becomes

$$
\frac{2\left(x e^{t}\right)^{-\alpha / 2}}{e^{t}-1} \exp \left(\frac{x e^{t}}{e^{t}-1}\right) \int_{0}^{\infty} \exp \left(-\frac{y^{2}}{e^{t}-1}\right) J_{\alpha}\left(\frac{2 \sqrt{x e^{t}}}{e^{t}-1} y\right) y^{\alpha+1} L_{n}^{\alpha}\left(-y^{2}\right) d y .
$$

an integral given in 8.9(5) of [7]. Simplifying the result of the computation, we obtain the left side of (3.8).

4. Formal considerations. One of our primary goals is the determination of an inversion operator for the dual Weierstrass-Laguerre transform. To this end, we seek, formally, to derive such an operator.

We know that, within the region of convergence of the integral, the dual Poisson-Laguerre transform

$$
u(x, t)=\int_{0}^{\infty} g_{\alpha}(x, y ; t) \phi(y) d \Lambda_{\alpha}(y)
$$

represents a dual Laguerre temperature with initial temperature $u\left(x, 0^{+}\right)=\phi(x)$. Let us, formally, consider the dual Weierstrass-Laguerre transform

$$
f(x)=\int_{0}^{\infty} g_{\alpha}(x, y ; 1) \phi(y) d \Lambda_{\alpha}(y)
$$

as

$$
f(x)=u(x, 1)
$$

Now $u(x, t)$ may be formally represented by

$$
u(x, t)=e^{t \nabla_{x}} \phi(x) .
$$

For, if we regard $\nabla_{x}$ as a number, it follows that

$$
\frac{\partial}{\partial t} u(x, t)=\nabla_{x} e^{t \nabla_{x}} \phi(x)=\nabla_{x} u(x, t)
$$

so that the Laguerre differential heat equation (2.8) is satisfied, and letting $t \rightarrow 0^{+}$in (4.4), we have $u\left(x, 0^{+}\right)=\phi(x)$.

Taking note of (4.3), we derive from (4.4) for $t=1$, treating $\nabla_{x}$ as a number,

$$
e^{-\nabla_{x}} f(x)=\phi(x)
$$

as the inversion formula for the dual Weierstrass-Laguerre transform (4.2). 
Our object, thus, is to obtain a reasonable definition for the operator $e^{-\nabla_{x}}$. To this end, we proceed, formally, by setting

$$
e^{-t \nabla_{x}}=\sum_{k=0}^{\infty} \frac{\left(-t \nabla_{k}\right)^{k}}{k !} .
$$

Appealing to (4.6) and taking note of (2.6), we have

$$
e^{-t \nabla_{x}} L_{n}^{\alpha}(x)=\sum_{k=0}^{\infty} \frac{(t n)^{k}}{k !} L_{n}^{\alpha}(x)=e^{t n} L_{n}^{\alpha}(x) .
$$

Now, let

$$
f(x)=\sum_{n=0}^{\infty} L_{n}^{\alpha}(x) a(n) \rho_{\alpha}(n)
$$

so that, referring to (2.1) and (2.3), we have

$$
a(n)=\int_{0}^{\infty} L_{n}^{\alpha}(x) f(x) d \Omega_{\alpha}(x) .
$$

Applying the operator $e^{-t \nabla_{x}}$ to (4.8), we find that

$$
\begin{aligned}
e^{-t \nabla_{x}} f(x) & =\sum_{n=0}^{\infty} e^{-t \nabla_{x}} L_{n}^{\alpha}(x) a(n) \rho_{\alpha}(n) \\
& =\sum_{n=0}^{\infty} e^{t n} L_{n}^{\alpha}(x) a(n) \rho_{\alpha}(n) \\
& =\sum_{n=0}^{\infty} a(n) \rho_{\alpha}(n) \int_{0}^{\infty} e^{-t(\alpha+1)} e^{x+y} g_{\alpha}(-x, y ; t) L_{n}^{\alpha}(-y) d \Lambda_{\alpha}(y) \\
& =\int_{0}^{\infty} e^{-t(\alpha+1)} e^{x+y} g_{\alpha}(-x, y ; t) d \Lambda_{\alpha}(y) \sum_{n=0}^{\infty} L_{n}^{\alpha}(-\dot{y}) a(n) \rho_{\alpha}(n) \\
& =\int_{0}^{\infty} e^{-t(\alpha+1)} e^{x+y} f(-y) g_{\alpha}(-x, y ; t) d \Lambda_{\alpha}(y),
\end{aligned}
$$

where we have appealed, respectively, to (4.7), (3.8), and (4.8), and have carried out the computations formally.

We thus define the operator $e^{-\nabla_{x}}$ as follows.

$$
e^{-\nabla_{x}} f(x)=\lim _{t \rightarrow 1^{-}} \int_{0}^{\infty} e^{-t(\alpha+1)} e^{x+y} f(-y) g_{\alpha}(-x, y ; t) d \Lambda_{\alpha}(y) .
$$

That the operator inverts the dual Weierstrass-Laguerre transform may now be shown, formally, as follows. We have

$$
f(x)=\int_{0}^{\infty} g_{\alpha}(x, y ; 1) \phi(y) d \Lambda_{\alpha}(y)
$$

so that

$$
f(-x)=\int_{0}^{\infty} g_{\alpha}(-x, y ; 1) \phi(y) d \Lambda_{\alpha}(y)
$$


Now, by (4.11),

$$
\begin{aligned}
e^{-\nabla_{x}} f(x) & =\lim _{t \rightarrow 1^{-}} \int_{0}^{\infty} e^{-t(\alpha+1)} e^{x+y} f(-y) g_{\alpha}(-x, y ; t) d \Lambda_{\alpha}(y) \\
& =\lim _{t \rightarrow 1^{-}} \int_{0}^{\infty} e^{-t(\alpha+1)} e^{x+y} g_{\alpha}(-x, y ; t) d \Lambda_{\alpha}(y) \\
& \int_{0}^{\infty} g_{\alpha}(-y, z ; 1) \phi(z) d \Lambda_{\alpha}(z) \\
= & \lim _{t \rightarrow 1^{-}} \int_{0}^{\infty} \phi(z) d \Lambda_{\alpha}(z) \int_{0}^{\infty} e^{-t(\alpha+1)} e^{x+y} \\
& \cdot g_{\alpha}(-x, y ; t) g_{\alpha}(-y, z ; 1) d \Lambda_{\alpha}(y) \\
= & \lim _{t \rightarrow 1^{-}} \int_{0}^{\infty} g_{\alpha}(x, z ; 1-t) \phi(z) d \Lambda_{\alpha}(z)=\phi(x)
\end{aligned}
$$

where we have invoked, respectively, (4.13) and (3.6), and have used, in the last step, the fundamental inversion theorem for the dual Poisson-Laguerre transform referred to in the second paragraph of this section.

The formal computation thus yields the inversion formula to be rigorously established.

5. Class H. The exponential operator applied to a function of suitable growth generates a dual Laguerre temperature as established in the following theorem.

THEOREM 5.1. Let a function $f$ defined for $0 \leqslant x<\infty$ satisfy the condition

$$
f(-y)=O\left(e^{y /(e-1)}\right), \quad y \rightarrow \infty .
$$

Then

$$
\begin{aligned}
u(x, t) & =e^{-(1-t) \nabla_{x}} f(x) \\
& =e^{-(1-t)(\alpha+1)} e^{x} \int_{0}^{\infty} e^{y} g_{\alpha}(-x, y ; 1-t) f(-y) d \Lambda_{\alpha}(y)
\end{aligned}
$$

belongs to class $\mathrm{H}$ for $0<t<1$.

Proof. We have

$$
\begin{aligned}
\nabla_{x} u(x, t) & =\int_{0}^{\infty} \nabla_{x}\left[e^{-(1-t)(\alpha+1)} e^{x+y} g_{\alpha}(-x, y ; 1-t) f(-y)\right] d \Lambda_{\alpha}(y) \\
& =\int_{0}^{\infty} \frac{\partial}{\partial t}\left[e^{-(1-t)(\alpha+1)} e^{x+y} g_{\alpha}(-x, y ; 1-t) f(-y)\right] d \Lambda_{\alpha}(y) \\
& =\frac{\partial}{\partial t} u(x, t)
\end{aligned}
$$

where differentiation under the integral sign is valid provided that the final integral converges uniformly for $0 \leqslant x<R, \delta \leqslant t \leqslant 1-\delta$ with $0<\delta<1$. That this is so is a consequence of the fact that for $0 \leqslant x<R, \delta \leqslant t \leqslant 1-\delta$, using (3.1), (3.4), and 
the fact that $|\mathscr{J}(z)|<1$, we have

$$
\begin{aligned}
& \begin{array}{l}
\int_{0}^{\infty}\left[\frac{\partial}{\partial t} e^{-(1-t)(\alpha+1)} e^{x+y} g_{\alpha}(-x, y ; 1-t) f(-y)\right] d \Lambda_{\alpha}(y) \mid \\
\leqslant \mid \int_{0}^{\infty} e^{-(1-t)(\alpha+1)} e^{x}\left\{\left[(\alpha+1)\left(1-\frac{1}{e^{1-t}-1}\right)-\frac{(x-y) e^{1-t}}{\left(e^{1-t}-1\right)^{2}}\right] \mid g_{\alpha}(-x, y ; 1-t)\right. \\
\left.\quad+\frac{x y}{\alpha+1} \frac{e^{1-t}+1}{\left(e^{1-t}-1\right)^{2}} g_{\alpha+1}(-x, y ; 1-t)\right\} f(-y) d \Lambda_{\alpha}(y) \\
\quad+(x+y) \frac{e^{1-t}}{\left(e^{1-t}-1\right)^{2}} \\
\left.\quad+\frac{x y}{\alpha+1} \frac{e^{1-t}+1}{\left(e^{1-t}-1\right)^{2}} \frac{e^{1-t}}{\left(e^{1-t}-1\right)}\right\}|f(-y)| d \Lambda_{\alpha}(y) \\
\leqslant \int_{0}^{\infty} e^{\left(e^{1-t} /\left(e^{1-t}-1\right)\right) x} \frac{e^{-y /\left(e^{1-t}-1\right)}}{\left(e^{1-t}-1\right)^{\alpha+1}}\left\{(\alpha+1)\left|1-\frac{1}{e^{1-t}-1}\right|\right. \\
\leqslant A(x, \delta) \int_{0}^{\infty} e^{-y /\left(e^{1-\delta}-1\right)}\left\{(\alpha+1)\left|e^{1-\delta}-2\right|+(R+y) \frac{e^{1-\delta}}{e^{\delta}-1}\right. \\
\left.+\frac{R y\left(e^{1-\delta}+1\right) e^{1-\delta}}{\left(e^{\delta}-1\right)^{2}}\right\}|f(-y)| d \Lambda_{\alpha}(y) .
\end{array}
\end{aligned}
$$

The dominant integral is independent of $x$ and $t$ and converges. Hence the result follows.

6. Inversion. We shall need, in our development, the fundamental inversion theorem for the dual Poisson-Laguerre transform referred to earlier, established in [5], and stated fully as follows.

THEOREM 6.1. Let $\phi$ be integrable in every finite interval, and let

$$
\int_{0}^{\infty} g_{\alpha}(x, y ; 1) \phi(y) d \Lambda_{\alpha}(y)
$$

converge for some $x=x_{0} \geqslant 0$. If

$$
\lim _{h \rightarrow 0} \frac{1}{h} \int_{x}^{x+h}[\phi(y)-\phi(x)] d \Lambda_{\alpha}(y)=0,
$$

then

$$
\lim _{t \rightarrow 0^{+}} \int_{0}^{\infty} g_{\alpha}(x, y ; t) \phi(y) d \Lambda_{\alpha}(y)=\phi(x) .
$$

Further, our principal inversion formula will be based on the following result.

THEOREM 6.2. Let $\beta$ be a function of bounded variation in every finite subinterval of $0 \leqslant x<\infty$. If the dual Weierstrass-Laguerre-Stieltjes transform

$$
f(x)=\int_{0}^{\infty} g_{\alpha}(x, y ; 1) d \beta(y)
$$

converges for $0 \leqslant x<\infty$, then, for $0<t<1,0 \leqslant x<\infty$,

$$
e^{-t \nabla_{x}} f(x)=\int_{0}^{\infty} g_{\alpha}(x, y ; 1-t) d \beta(y) .
$$


Proof. From Theorem 6.2 of [5], we know that

$$
\beta(y)=o\left(\frac{1}{g_{\alpha}(x, y ; t)}\right), \quad y \rightarrow \infty .
$$

and that $\beta\left(0^{+}\right)$exists. Further, by Theorem 5.2 of [5], the integral in (6.4) converges for $x$ negative and we have, on integrating by parts,

$$
\begin{aligned}
f(-x) & =\int_{0}^{\infty} g_{\alpha}(-x, y ; 1) d \beta(y) \\
& =\left.g_{\alpha}(-x, y ; 1) \beta(y)\right|_{0} ^{\infty}-\int_{0}^{\infty} \beta(y) \frac{\partial}{\partial y} g_{\alpha}(-x, y ; 1) d y \\
& =-g_{\alpha}(-x ; 1) \beta\left(0^{+}\right)-\int_{0}^{\infty} \beta(y) \frac{\partial}{\partial y} g_{\alpha}(-x, y ; 1) d y .
\end{aligned}
$$

Hence

$$
\begin{aligned}
e^{-t \nabla_{x}} f(x)= & \int_{0}^{\infty} e^{-t(\alpha+1)} e^{x+y} f(-y) g_{\alpha}(-x, y ; t) d \Lambda_{\alpha}(y) \\
= & \int_{0}^{\infty} e^{-t(\alpha+1)} e^{x+y} g_{\alpha}(-x, y ; t) \\
& \cdot\left[-g_{\alpha}(-y ; 1) \beta\left(0^{+}\right)-\int_{0}^{\infty} \beta(z) \frac{\partial}{\partial z} g_{\alpha}(-y, z ; 1) d z\right] d \Lambda_{\alpha}(y) \\
= & -\beta\left(0^{+}\right) g_{\alpha}(x ; 1-t)-\int_{0}^{\infty} e^{-t(\alpha+1)} e^{x+y} g_{\alpha}(-x, y ; t) d \Lambda_{\alpha}(y) \\
& \cdot \int_{0}^{\infty} \beta(z) \frac{\partial}{\partial z} g_{\alpha}(-y, z ; 1) d z,
\end{aligned}
$$

or, applying (3.4) to the integrand on the right, we obtain

$$
\begin{aligned}
e^{-t \nabla_{x}} f(x)= & -\beta\left(0^{+}\right) g_{\alpha}(x ; 1-t)-\int_{0}^{\infty} e^{-t(\alpha+1)} e^{x+y} g_{\alpha}(-x, y ; t) d \Lambda_{\alpha}(y) \\
& \cdot \int_{0}^{\infty} \frac{\beta(z)}{e-1}\left[-\frac{x}{\alpha+1} g_{\alpha+1}(-y, z ; 1)-g_{\alpha}(-y, z ; 1)\right] d z
\end{aligned}
$$

Now, the integral on the right is dominated by

$$
A(x, t) \int_{0}^{\infty} e^{-y /\left(e^{t}-1\right)} y^{\alpha} d y \int_{0}^{\infty}|\beta(z)|\left[\frac{|x|}{(\alpha+1)}\left(\frac{e}{e-1}\right)+1\right] e^{(y-z) /(e-1)} d z
$$

which, because of (6.5) converges absolutely. Fubini's theorem is thus applicable and we have

$$
\begin{aligned}
e^{-t \nabla_{x}} f(x)= & -\beta\left(0^{+}\right) g_{\alpha}(x ; 1-t)-\int_{0}^{\infty} \beta(z) \\
& \cdot\left[\frac{\partial}{\partial z} \int_{0}^{\infty} e^{-t(\alpha+1)} e^{x+y} g_{\alpha}(-x, y ; t) g_{\alpha}(-y, z ; 1) d \Lambda_{\alpha}(y)\right] d z .
\end{aligned}
$$

Appealing to (3.6), we find that

$$
e^{-t \nabla_{x}} f(x)=-\beta\left(0^{+}\right) g_{\alpha}(x ; 1-t)-\int_{0}^{\infty} \beta(z) \frac{\partial}{\partial z} g_{\alpha}(x, z ; 1-t) d z .
$$


Integrating by parts again, we have

$$
\begin{aligned}
e^{-t \nabla_{x}} f(x)= & -\beta\left(0^{+}\right) g_{\alpha}(x ; 1-t)-\left.\beta(z) g_{\alpha}(x, z ; 1-t)\right|_{0} ^{\infty} \\
& +\int_{0}^{\infty} g_{\alpha}(x, z ; 1-t) d \beta(z) \\
= & \int_{0}^{\infty} g_{\alpha}(x, z ; 1-t) d \beta(z)
\end{aligned}
$$

We now prove our principal inversion formula.

THEOREM 6.3. Let $\phi(x)$ be integrable in every finite interval and let the dual Weierstrass-Laguerre transform

$$
f(x)=\int_{0}^{\infty} g_{\alpha}(x, y ; 1) \phi(y) d \Lambda_{\alpha}(y)
$$

converge. If

$$
\lim _{h \rightarrow 0^{+}} \frac{1}{h} \int_{x}^{x+h}[\phi(y)-\phi(x)] d \Lambda_{\alpha}(y)=0,
$$

then

$$
\lim _{t \rightarrow 1^{-}} e^{-t \nabla_{x}} f(x)=\phi(x) .
$$

PrOOF. From the preceding theorem, we have that

$$
e^{-\nabla_{x}} f(X)=\int_{0}^{\infty} g_{\alpha}(x, y ; 1-t) \phi(y) d \Lambda_{\alpha}(y) .
$$

The result then follows on applying the fundamental inversion Theorem 6.1.

7. Representation. We seek to characterize those functions which may be represented by dual Weierstrass-Laguerre transforms of increasing functions. To this end, we shall need to invoke the following basic theorem established in [5].

THEOREM 7.1. A necessary and sufficient condition that

$$
u(x, t)=\int_{0}^{\infty} g_{\alpha}(x, y ; t) d \beta(y), \quad 0 \leqslant x<\infty,
$$

with $\beta(y) \uparrow$ and the integral converging for $0<t<c$ for some real number $c$ is that $u(x, t)$ be a nonnegative dual Laguerre temperature.

Our principal result may now be proved.

THEOREM 7.2. Let $f$ be a function defined for $0 \leqslant x<\infty$ and satisfying the inequality

$$
e^{-t \nabla_{x}} f(x) \geqslant 0,
$$

and let its associated function $f(x,-y)$ satisfy the conditions

$$
\begin{gathered}
f(x,-y)=O\left(e^{y /(e-1)}\right), \quad y \rightarrow \infty, \\
\frac{\partial}{\partial y} f(x,-y)=O\left(e^{y /(e-1)}\right), \quad y \rightarrow \infty .
\end{gathered}
$$


Then $f$ has the dual Weierstrass-Laguerre transform representation

$$
f(x)=\int_{0}^{\infty} g_{\alpha}(x, y, 1) d \beta(y),
$$

with $\beta(y) \uparrow$.

Proof. Let

$$
u(x, t)=e^{-(1-t) \nabla_{x}} f(x) .
$$

By definition (4.10) and condition (7.3), we note that $u(x, t)$ is well defined. Since $u(x, t)$ is nonnegative by (7.2) and belongs to class $H$ by Theorem 5.1 , it satisfies the hypotheses of Theorem 7.1 and we have that

$$
u(x, t)=\int_{0}^{\infty} g_{\alpha}(x, y ; t) d \beta(y), \quad \beta(y) \uparrow .
$$

Now, using (4.10), we have

$$
u(x, t)=e^{-(1-t)(\alpha+1)} e^{x} \int_{0}^{\infty} e^{y} f(-y) g_{\alpha}(-x, y ; 1-t) d \Lambda_{\alpha}(y) .
$$

It then follows that

$$
\begin{gathered}
\nabla_{x} u(x, t)=e^{-(1-t)(\alpha+1)} \int_{0}^{\infty} e^{y} f(-y) \nabla_{x} e^{x} g_{\alpha}(-x, y ; 1-t) d \Lambda_{\alpha}(y) \\
=e^{-(1-t)(\alpha+1)} e^{x} \int_{0}^{\infty} e^{y} f(-y) \mid \nabla_{x} g_{\alpha}(-x, y ; 1-t) \\
+2 x \frac{\partial}{\partial x} g_{\alpha}(-x, y ; 1-t) \\
+(\alpha+1) g_{\alpha}(-x, y, 1-t) \mid d \Lambda_{\alpha}(y) \\
=e^{-(1-t)(\alpha+1)} e^{x} \int_{0}^{\infty} e^{y} f(-y) \mid-\nabla_{y} g_{\alpha}(-x, y ; 1-t) \\
\quad+(\alpha+1) g_{\alpha}(-x, y ; 1-t) \mid d \Lambda_{\alpha}(y) \\
=-e^{-(1-t)(\alpha+1)} e^{x} \int_{0}^{\infty} e^{y} f(-y) \nabla_{y} g_{\alpha}(-x, y ; 1-t) d \Lambda_{\alpha}(y)+(\alpha+1) u(x, t),
\end{gathered}
$$

where the validity of the differentiation under the integral sign is established as in Theorem 5.1. We thus have that

$$
\begin{aligned}
\left.\nabla_{x} u(x, t)\right|_{x=0}= & -e^{-(1-t)(\alpha+1)} \int_{0}^{\infty} e^{y} f(-y) \nabla_{y} g_{\alpha}(y ; 1-t) d \Lambda_{\alpha}(y) \\
& +(\alpha+1) u(0, t) .
\end{aligned}
$$

Now let us set

$$
\begin{aligned}
v(x, t) & =e^{-(1-t)(\alpha+1)} \int_{0}^{\infty} e^{y} f(x,-y) g_{\alpha}(y ; 1-t) d \Lambda_{\alpha}(y) \\
& =\frac{e^{-(1-t)(\alpha+1)}}{\Gamma(\alpha+1)} \int_{0}^{\infty} y^{\alpha} f(x,-y) g_{\alpha}(y ; 1-t) d y
\end{aligned}
$$


It follows that

$$
\begin{aligned}
\nabla_{x} v(x, t) & =\frac{e^{-(1-t)(\alpha+1)}}{\Gamma(\alpha+1)} \int_{0}^{\infty} y^{\alpha}\left[\nabla_{x} f(x,-y)\right] g_{\alpha}(y ; 1-t) d y \\
& =\frac{e^{-(1-t)(\alpha+1)}}{\Gamma(\alpha+1)} \int_{0}^{\infty} y^{\alpha}\left[-\nabla_{y} f(x,-y)-2 y \frac{\partial}{\partial y} f(x,-y)\right] g_{\alpha}(y ; 1-t) d y \\
& =-\frac{e^{-(1-t)(\alpha+1)}}{\Gamma(\alpha+1)} \int_{0}^{\infty} e^{-y} \frac{\partial}{\partial y}\left[y^{\alpha+1} e^{y} \frac{\partial}{\partial y} f(x,-y)\right] g_{\alpha}(y ; 1-t) d y \\
& =-\frac{e^{-(1-t)(\alpha+1)}}{\Gamma(\alpha+1)}\left\{\left.e^{-y} g_{\alpha}(y ; 1-t) y^{\alpha+1} e^{y} \frac{\partial}{\partial y} f(x,-y)\right|_{0} ^{\infty}\right. \\
& \left.-\int_{0}^{\infty}\left[y^{\alpha+1} e^{y} \frac{\partial}{\partial y} f(x,-y)\right] \frac{\partial}{\partial y}\left[e^{-y} g_{\alpha}(y ; 1-t)\right] d y\right\},
\end{aligned}
$$

where the validity of the differentiation under the integral sign follows from condition (7.3) which also results in the vanishing of the integrated part of the integration by parts of the last step. Integrating by parts again, we have

$$
\begin{aligned}
\nabla_{x} v(x, t)=\frac{e^{-(1-t)(\alpha+1)}}{\Gamma(\alpha+1)}\{ & \left.f(x,-y) y^{\alpha+1} e^{y} \frac{\partial}{\partial y}\left[e^{-y} g_{\alpha}(y ; 1-t)\right]\right|_{0} ^{\infty} \\
& \left.-\int_{0}^{\infty} f(x,-y) \frac{\partial}{\partial y}\left[y^{\alpha+1} e^{y} \frac{\partial}{\partial y} e^{-y} g_{\alpha}(y ; 1-t)\right] d y\right\} .
\end{aligned}
$$

Again, by (7.3), the integrated part vanishes and we have

$$
\begin{aligned}
& \nabla_{x} v(x, t)=-\frac{e^{-(1-t)(\alpha+1)}}{\Gamma(\alpha+1)} \int_{0}^{\infty} f(x,-y) \frac{\partial}{\partial y}\left[y^{\alpha+1} e^{y} \frac{\partial}{\partial y} g_{\alpha}(y ; 1-t)\right] d y \\
& =-e^{-(1-t)(\alpha+1)} \int_{0}^{\infty} e^{y} y^{-\alpha} f(x,-y) \frac{\partial}{\partial y}\left[y^{\alpha+1} e^{y} \frac{\partial}{\partial y} g_{\alpha}(y ; 1-t)\right] d \Lambda_{\alpha}(y) \\
& =-e^{-(1-t)(\alpha+1)} \int_{0}^{\infty} e^{y} f(x,-y)\left[\nabla_{y} g_{\alpha}(y ; 1-t)-(\alpha+1) g_{\alpha}(y ; 1-t)\right] d \Lambda_{\alpha}(y) \\
& =-e^{-(1-t)(\alpha+1)} \int_{0}^{\infty} e^{y} f(x,-y) \nabla_{y} g_{\alpha}(y ; 1-t) d \Lambda_{\alpha}(y)+(\alpha+1) v(x, t) .
\end{aligned}
$$

Hence

$$
\begin{aligned}
\left.\nabla_{x} v(x, t)\right|_{x=0}= & -e^{-(1-t)(\alpha+1)} \int_{0}^{\infty} e^{y} f(-y) \nabla_{y} g_{\alpha}(y ; 1-t) d \Lambda_{\alpha}(y) \\
& +(\alpha+1) v(0, t) .
\end{aligned}
$$

We note from (7.8) and (7.11) that $u(0, t)=v(0, t)$. It follows from (7.10) and (7.13) that

$$
\left.\nabla_{x} u(x, t)\right|_{x=0}=\left.\nabla_{x} v(x, t)\right|_{x=0} .
$$

Moreover, by induction, we may establish that

$$
\left.\nabla_{x}^{n} u(x, t)\right|_{x=0}=\left.\nabla_{x}^{n}(x, t)\right|_{x=0}, \quad n=0,1,2, \ldots
$$


But, from the definition of $\nabla_{x}$, it is clear that

$$
\left.\nabla_{x}^{n} u(x, t)\right|_{x=0}=\left.(\alpha+1) \frac{\partial^{n} u}{\partial x^{n}}\right|_{x=0}=\left.(\alpha+1) \frac{\partial^{n} v}{\partial x^{n}}\right|_{x=0}
$$

so that the Maclaurin coefficients for the functions $u(x, t)$ and $v(x, t)$ are identical. Hence $u(x, t)=v(x, t), 0 \leqslant x<\infty$.

We thus have

$$
\begin{aligned}
u(x, t) & =e^{-(1-t)(\alpha+1)} \int_{0}^{\infty} f(x,-y) g_{\alpha}(y ; 1-t) y^{\alpha} \frac{d y}{\Gamma(\alpha+1)} \\
& =\frac{1}{\left(e^{1-t}-1\right)^{\alpha+1}} \int_{0}^{\infty} f(x,-y) \exp \left(-\frac{y}{e^{1-t}-1}\right) y^{\alpha} \frac{d y}{\Gamma(\alpha+1)}
\end{aligned}
$$

or, by a change of variables,

$$
\begin{aligned}
u(x, t)= & \frac{1}{\left(e^{1-t}-1\right)^{\alpha+1}} \int_{0}^{\infty} f\left(x,-y \frac{e^{1-t}-1}{e-1}\right) e^{-y /(e-1)} \\
& \cdot y^{\alpha}\left(\frac{e^{1-t}-1}{e-1}\right)^{\alpha+1} \frac{d y}{\Gamma(\alpha+1)} \\
= & e^{-(\alpha+1)} \int_{0}^{\infty} e^{y} f\left(x,-y \frac{e^{1-t}-1}{e-1}\right) g_{\alpha}(y ; 1) d \Lambda_{\alpha}(y) .
\end{aligned}
$$

Appealing to (7.3), we note that the integral is dominated by

$$
\begin{aligned}
A \int_{0}^{\infty} \exp \left(y \frac{e^{1-t}-1}{e-1}\right) & \exp \left(-\frac{y}{e-1}\right) y^{\alpha} d y \\
& <A \int_{0}^{\infty} \exp \left(-\frac{e-e^{1-\delta}}{(e-1)^{2}}\right) y^{\alpha} d y, \quad \delta \leqslant t<1,
\end{aligned}
$$

a convergent integral independent of $t$. Lebesgue's dominating convergence theorem thus applies and

$$
u(x, 1-)=e^{-(\alpha+1)} f(x) \int_{0}^{\infty} e^{y} g_{\alpha}(y ; t) d \Lambda_{\alpha}(y)=f(x) .
$$

Now, for $R>0$, we note from (7.7), since the integrand is nonnegative and $\beta(y) \uparrow$, that

$$
u(x, t) \geqslant \int_{0}^{R} g_{\alpha}(x, y ; t) d \beta(y), \quad 0<t<1,0 \leqslant x<\infty .
$$

But

$$
\begin{aligned}
\int_{0}^{R} g_{\alpha}(x, y ; t) d \beta(y) & \leqslant K\left(\frac{e^{t}}{e^{t}-1}\right)^{\alpha+1} \int_{0}^{R} \exp \left(-\frac{(\sqrt{x}-\sqrt{y e})^{2}}{e^{t}-1}\right) d \beta(y) \\
& \leqslant K\left(\frac{e}{e^{\delta}-1}\right)^{\alpha+1} \int_{0}^{R} d \beta(y), \quad 0<\delta \leqslant t<1
\end{aligned}
$$


with the dominating integral converging. From (7.15) and (7.16) we then have that

$$
f(x) \geqslant \int_{0}^{R} g_{\alpha}(x, y ; 1) d \beta(y), \quad 0 \leqslant x<\infty .
$$

It follows that $\int_{0}^{\infty} g_{\alpha}(x, y ; t) d \beta(y)$ converges, and by Theorem 5.1 of [5] that

$$
\lim _{t \rightarrow 1} \int_{0}^{\infty} g_{\alpha}(x, y ; t) d \beta(y)=\int_{0}^{\infty} g_{\alpha}(x, y ; 1) d \beta(y)
$$

or

$$
f(x)=\int_{0}^{\infty} g_{\alpha}(x, y ; 1) d \beta(y)
$$

and the representation is established.

Of the sufficient conditions of the theorem, we show that condition (7.2) is necessary for the representation (7.5).

THEOREM 7.3. If

$$
f(x)=\int_{0}^{\infty} g_{\alpha}(x, y ; 1) d \beta(y), \quad \beta(y) \uparrow,
$$

then $e^{-t \nabla_{x}} f(x) \geqslant 0$.

Proof. We have that

$$
\begin{aligned}
e^{-t \nabla_{x}} f(x) & =e^{-t(\alpha+1)} e^{x} \int_{0}^{\infty} e^{y} f(-y) g_{\alpha}(-x, y ; t) d \Lambda_{\alpha}(y) \\
& =e^{-t(\alpha+1)} e^{x} \int_{0}^{\infty} g(-x, y ; t) d \Lambda_{\alpha}(y) \int_{0}^{\infty} g(-y, z ; 1) d \beta(z) .
\end{aligned}
$$

The integrated integral is dominated by

$$
K(x, t) \int_{0}^{\infty} \exp \left(-\frac{y}{e^{t}-1}+\frac{y}{e-1}\right) y^{\alpha} d y \int_{0}^{\infty} g_{\alpha}(z ; 1) d \beta(z)<\infty,
$$

so that Fubini's theorem is applicable and we may interchange the order of integration. We then have

$$
e^{-t \nabla_{x}} f(x)=e^{-t(\alpha+1)} e^{x} \int_{0}^{\infty} d \beta(z) \int_{0}^{\infty} e^{y} g_{\alpha}(-x, y ; t) g_{\alpha}(-y, z ; 1) d \Lambda_{\alpha}(y) .
$$

On appealing to (3.6), we find that

$$
e^{-t \nabla_{x}} f(x)=\int_{0}^{\infty} g_{\alpha}(x, z, 1-t) d \beta(z) .
$$

Since our kernel is nonnegative and $\beta(z) \uparrow$, the result follows.

The derivation of suitably sensitive estimates for $d(x,-y, z)$ is indicated for attempting to resolve the question of the necessity of conditions (7.3) and (7.4).

8. Examples. The validity of the inversion algorithm may be illustrated by the example

$$
g_{\alpha}(x ; a), \quad a>1,
$$

which is the dual Weierstrass-Laguerre transform

$$
g_{\alpha}(x ; a)=\int_{0}^{\infty} g_{\alpha}(x, y ; 1) g_{\alpha}(y ; a-1) d \Lambda_{\alpha}(y), \quad a>1 .
$$


We have that

$$
\begin{aligned}
e^{-\nabla_{x}} g_{\alpha}(x ; a) & =\lim _{t \rightarrow 1^{-}} e^{-(\alpha+1) t} e^{x} \int_{0}^{\infty} e^{y} g_{\alpha}(-y ; a) g_{\alpha}(-x, y ; t) d \Lambda_{\alpha}(y) \\
& =g(x ; a-1)
\end{aligned}
$$

by (3.6) as predicted by Theorem 6.3.

The Laguerre heat polynomials $p_{n, \alpha}(x, 1)$ of [6] given by

$$
p_{n, \alpha}(x, 1)=\sum_{k=0}^{n} \frac{\Gamma(n+\alpha+1)}{\Gamma(k+\alpha+1)}\left(\begin{array}{l}
n \\
k
\end{array}\right)\left(1-e^{-1}\right)^{n-k}\left(x e^{-1}\right) k,
$$

with the integral representation

$$
p_{n, \alpha}(x, 1)=\int_{0}^{\infty} g_{\alpha}(x, y ; 1) y^{n} d \Lambda_{\alpha}(y), \quad n=0,1,2, \ldots,
$$

provide another example of the inversion algorithm. The inversion will be established if

$$
e^{-\nabla_{x}} p_{n, \alpha}(x, 1)=x^{n}, \quad n=0,1,2, \ldots
$$

or

$$
x^{n}=\lim _{t \rightarrow 1^{-}} e^{-(\alpha+1) t} e^{x} \int_{0}^{\infty} e^{y} p_{n, \alpha}(-y, 1) g_{\alpha}(-x, y ; t) d \Lambda_{\alpha}(y) .
$$

Now, by p. 290, (2.13) and p. 289, (2.8) of [6], we have

$$
x^{n}=\int_{0}^{\infty}\left(-e^{t}\right)^{n} p_{n, \alpha}\left(-z e^{t}, t\right) g_{\alpha}(x, z ; t) d \Lambda_{\alpha}(z) .
$$

Replacing $x$ by $-x e^{t}$, making the substitution $z=y e^{-t}$, and noticing that

$$
g_{\alpha}\left(-x e^{t}, y e^{-t} ; t\right)=e^{x+y e^{-t}} g_{\alpha}(-x, y ; t),
$$

we have

$$
\left(-x e^{t}\right)^{n}=\left(-e^{t}\right)^{n} \int_{0}^{\infty} e^{x+y e^{-t}} g_{\alpha}(-x, y ; t) p_{n, \alpha}(-y, t) \frac{e^{y-y e^{-t}}}{\left(e^{t}\right)^{\alpha+1}} d \Lambda_{\alpha}(y)
$$

The inversion is established on letting $t \rightarrow 1^{-}$.

Each of the previous examples serves to illustrate the representation theorem as well.

For the function $g_{\alpha}(x ; a), a>0$, we have that

$$
\begin{aligned}
e^{-t \nabla_{x}} g_{\alpha}(x ; a) & =g_{\alpha}(x ; a-t), \quad 0<t<1, \\
& \geqslant 0
\end{aligned}
$$

Moreover, since

$$
g_{\alpha}(x,-y ; a)=\left(\frac{e^{a}}{e^{a}-1}\right)^{\alpha+1} \exp \left(-\frac{x}{e^{a}-1}+\frac{y}{e^{a-1}}\right) \mathscr{J}\left(\frac{2 \sqrt{x y e^{a}}}{e^{a}-1}\right)
$$

and

$$
\frac{\partial}{\partial y} g_{\alpha}(x,-y ; a)=\frac{g_{\alpha}(x,-y ; a)}{e^{a}-1}-\frac{x}{e^{a}-1} g_{\alpha+1}(x,-y, a),
$$


it follows that

$$
\begin{gathered}
g_{\alpha}(x,-y ; a)=O\left(e^{y /\left(e^{u}-1\right)}\right), \quad y \rightarrow \infty, \\
\frac{\partial}{\partial y} g_{\alpha}(x,-y ; a)=O\left(e^{y /\left(e^{u}-1\right)}\right), \quad y \rightarrow \infty .
\end{gathered}
$$

The conditions of Theorem 7.1 are met and the conclusion holds with the representation (8.2).

For the Laguerre heat polynomials we have that

$$
\begin{aligned}
e^{-t \nabla_{x}} p_{n, \alpha}(x ; 1) & =p_{n, \alpha}(x ; 1-t), \quad 0<t<1, \\
& \geqslant 0 .
\end{aligned}
$$

Moreover, for the associated functions, we have, on appealing to (8.4) and (2.15),

$$
\begin{aligned}
& p_{n, \alpha}(x,-y ; 1)=\sum_{k=0}^{n} \frac{\Gamma(n+\alpha+1)}{\Gamma(k+\alpha+1)}\left(\begin{array}{l}
n \\
k
\end{array}\right)\left(1-e^{-1}\right)^{n-k}\left(e^{-1}\right)^{k} \\
& \quad \cdot \int_{0}^{\infty} z^{k} d(x,-y, z) d \Lambda_{\alpha}(z) \\
&=\sum_{k=0}^{n} \frac{\Gamma(\alpha+1) \Gamma(n+\alpha+1) n !}{\Gamma(n-k) !}\left(1-e^{-1}\right)^{n-k}\left(-e^{-1}\right)^{k} \\
& \cdot \sum_{j=0}^{k} \frac{j !}{(k-j) ![\Gamma(j+\alpha+1)]^{2}} L_{j}^{\alpha}(x) L_{j}^{\alpha}(-y) \\
&= \Gamma(\alpha+1) \Gamma(n+\alpha+1) n !\left(1-e^{-1}\right)^{n} \sum_{j=0}^{n} \frac{j !}{[\Gamma(j+\alpha+1)]^{2}} L_{j}^{\alpha}(x) L_{j}^{\alpha}(-y) \\
& \cdot \sum_{k=j}^{n} \frac{(1-e)^{-k}}{(n-k) !(k-j) !},
\end{aligned}
$$

and, since $(d / d x) L_{n}^{\alpha}(x)=-L_{n-1}^{\alpha+1}(x)$, we have (8.14)

$$
\begin{aligned}
\frac{\partial}{\partial y} p_{n, \alpha}(x,-y ; 1)= & \Gamma(\alpha+1) \Gamma(n+\alpha+1) n !\left(1-e^{-1}\right)^{n} \\
& \cdot \sum_{j=0}^{n} \frac{j !}{[\Gamma(j+\alpha+1)]^{2}} L_{j}^{\alpha}(x) L_{j-1}^{\alpha+1}(-y) \sum_{k=j}^{n} \frac{(1-e)^{k}}{(n-k) !(k-j) !} .
\end{aligned}
$$

It is thus clear that

$$
p_{n, \alpha}(x,-y ; 1)=O\left(e^{y /(e-1)}\right), \quad y \rightarrow \infty,
$$

and

$$
\frac{\partial}{\partial y} p_{n, \alpha}(x,-y ; 1)=O\left(e^{y /(e-1)}\right), \quad y \rightarrow \infty,
$$

and the hypotheses of Theorem 7.1 are satisfied. The representation (8.5) thus gives the conclusion anticipated. 


\section{REFERENCES}

1. Frank M. Cholewinski and Deborah Tepper Haimo, The Weierstrass-Hankel convolution transform, J. Analyse Math. 17 (1966), 1-58.

2. L_ Laguerre temperatures, Proceedings of the Conference on Orthogonal Expansions and their Continuous Analogues, Southern Illinois Univ. Press, Carbondale, 1968, pp. 127-226,

3. _ The Poisson-Laguerre transform, Bull. Amer. Math. Soc. 74 (1968), 137-139.

4. _ The Weierstrass-Laguerre transform, J. Math. Anal. Appl. 23 (1968), 41-57.

5. __ The dual Poisson-Laguerre transform, Trans. Amer. Math. Soc. 144 (1969), 271-300.

6. __ Expansions in terms of Laguerre heat polynomials and their temperature traiisforms, J. Analyse Math. 24 (1971), 285-322.

7. A. Erdelyi, et al., Tables of integral transforms, vol. 2, McGraw-Hill, New York, 1954.

8. Deborah Tepper Haimo, Generalized temperature functions, Duke Math. J. 33 (1966), 305-322.

9. I. I. Hirschman and D. V. Widder, The convolution transform, Princeton Univ. Press, Princeton, N. J., 1955, pp. 173-209.

Department of Mathematical SCIENCES, UNiversity of MisSOURI - St. LoUis, St. LoUis, MisSOURI 63121 (Current address)

School of Mathematics, Institute for Advanced Study, Princeton, New Jersey 08540 